\title{
A Validated Questionnaire for Quantifying Skin Oiliness
}

\author{
Leslie S. Baumann1, Randall D. Penfield², Jennifer L. Clarke³, Deysi K. Duque \\ ${ }^{1}$ University of Miami Cosmetic Medicine and Research Institute, University of Miami, Miami Beach, USA \\ ${ }^{2}$ Department of Educational and Psychological Studies, University of Miami, Coral Gables, USA \\ ${ }^{3}$ Department of Epidemiology and Public Health, University of Miami, Miami, USA \\ ${ }^{4}$ Department of Clinical Research, Baumann Cosmetic \& Research Institute, Miami, USA \\ Email: Isb@derm.net
}

Received 29 January 2014; revised 25 February 2014; accepted 5 March 2014

Copyright (C) 2014 by authors and Scientific Research Publishing Inc.

This work is licensed under the Creative Commons Attribution International License (CC BY).

http://creativecommons.org/licenses/by/4.0/

\section{(c) (i) Open Access}

\begin{abstract}
Increased sebum production is a common skin complaint and plays an important role in acne and oily scalp conditions. To choose the correct skin care products, which mostly are marketed for dry, oily or normal skin, the consumer must self-assess their skin type. Studies show that individuals incorrectly self-assess their sebum secretion levels. In order to be able to correctly determine skin oiliness, we have developed a six-item skin oiliness scale (SOS) that correlates with sebumeter measurements. The resulting correlation was 0.54 , which was significantly different from zero ( $p$ $<0.01$ ). This result indicates a strong relationship between the SOS scores and the associated sebumeter measurements. This is easy to administer questionnaire to accurately determine skin oiliness and can be useful in screening and recruiting patients for research trials, performing outcome research, and recommending skin care products and procedures. Our study shows that this skin oiliness scale is an accurate way to identify and quantify skin oiliness.
\end{abstract}

\section{Keywords}

Skin Oiliness Scale; Oily Facial Skin; Skin Oiliness Quantification; Measuring Skin Oiliness

\section{Introduction}

Increased sebum secretion is a common cosmetic complaint but, in addition, plays an important role in acne and oily scalp conditions. Skin care products are often marketed to three separate skin types: dry, oily or normal, leaving the consumer to determine whether their skin is oily, dry or normal. Several studies have shown that individuals incorrectly assess their sebum secretion levels [1]. 
One study enrolled 94 Korean women and asked them to rate their skin type as oily, dry or normal. The sebumeter was then used to measure sebum secretion on 4 sites of the face. The results showed that the subjective skin type did not match the amount of sebum secreted, and that subjects underestimated their skin's sebum production. In some cases, those who believed that their skin type was dry, actually had "oily skin" as determined by the sebumeter [2]. A subject's estimation of their skin type is subject to many biases, and seasonal variations in sebum secretion can confuse the issue [3]. We have developed a questionnaire that asks a series of historical and subjective questions that have been found to correlate with sebumeter measurements. These questions offer a method of determining skin oiliness that can be used to screen candidates for research studies or to provide skin care advice and recommendations.

The Baumann Skin Type Indicator (BSTI) consists of a questionnaire about 4 main factors of the skin: 1) oily vs. dry, 2) sensitive vs. resistant, 3) pigmented vs. non-pigmented, and 4) wrinkled vs. tight [4]. A numerical score is derived from the questionnaire that places an individual into a distinct skin classification within 16 skin types based on these four parameters. The primary objective of this study was to determine if the O (oily) section questions of the BSTI could successfully discriminate between individuals with high and low sebum secretion rates as determined by the sebumeter measurements.

\section{Materials and Methods}

\subsection{Instrument Development}

The development and validation of the SOS followed standard measurement procedures, as outlined in Crocker and Algina, and Osterlind [5] [6]. The construction of the SOS began with the development of an initial pool of items measuring skin oiliness. This pool of items was reviewed by an expert panel to determine whether the content of each item was appropriate for the measurement of oiliness. Based on the recommendations of the expert panel, the initial pool of items was revised accordingly to a preliminary pool of 11 items (Table 1). The number of response options for each item was either four or five, depending on the item. The pool of 11 items was pilot tested using a sample of 400 respondents.

Using the data obtained in the pilot testing, the SOS was calibrated using the partial credit model [7], which provided a model-based approach for the measurement of skin oiliness. The PCM approach was deemed preferable to a true-score model approach because of its utility in examining item quality and the measurement properties of the scale [8] [9]. The PCM calibration yielded adequate fit (weighted mean squared errors, or infit, ranged from 0.7 to 1.26). The correlation of each item to the resulting trait estimate for each person (i.e. pointbiserial correlation, which serves as a measure of item discrimination) ranged between 0.47 and 0.74 . While these results indicated that all items displayed acceptable measurement properties, consideration of the relative performance of the items (i.e. according to fit and discrimination) allowed the research team to select the items that displayed the strongest psychometric properties, and thus would lead to scores having the highest level of reliability and validity.

Based on the results of the initial pilot test described above, five items were selected from the initial pool of 11 items such that the selected items displayed the strongest measurement properties (discrimination, fit, and content representation). The five selected items, in addition to one newly created item (How often do you wash your face?), formed the final six-item SOS. The six items of the SOS are displayed in Table 2. The decision to use only six items was based on several factors: a) a relatively short scale was sought to increase the userfriendliness and utility of the SOS; b) through consideration of the PCM results of the initial pilot test, it was determined that six items would yield a reliability of approximately 0.8 ; and c) content review of the six items by an expert panel indicated that these items sufficiently covered the outcomes associated with skin oiliness, and thus provided initial evidence of validity for scores obtained from the six-item SOS. To obtain internal structure and criterion-related validity evidence [6] of the scores obtained from the six-item SOS, a validation study was conducted in which 100 individuals were administered the SOS along with six sebumeter measurements (three sebumeter measurements were taken 45 minutes and three sebumeter measurements were taken 60 minutes following face washing).

\subsection{Sample Population and Data Collection}

In the pilot testing of the initial bank of 11 items, 400 male and female study participants were recruited to participate in the survey between July and November, 2005 (Table 3). Participants were patients at the Dermatolo- 
Table 1. Pool of 11 questions about skin oiliness production.

\section{Baumann Skin Type Questionnaire}

1. After washing your face, don't apply any moisturizer, sunscreen, toner, powder or other products. Two to three hours later, look in a mirror under bright lights. Your forehead and cheeks feel or appear:
a) Very rough, flaky, or ashy
b) Tight
c) No reflection of light, and well hydrated
d) Shiny with reflection of bright light

2. In photos, your face appears shiny:
a) Never, or you've never noticed shine
b) Sometimes
c) Frequently
d) Always

3. Two to three hours after applying make-up foundation (also known as base) but no powder, your make-up appears:
a) Flaky or caked in wrinkles
b) Smooth
c) Shiny
d) Streaked and shiny
e) I do not wear facial foundation

4. When in a low humidity environment, if you don't use moisturizers or sunscreen, your facial skin:
a) Feels very dry, or cracks
b) Feels tight
c) Feels normal
d) Looks shiny or I never feel that I need moisturizer
e) Don't know

5. Look in a magnifying mirror. How many large pores, the size of the end of a pin or greater, do you have?
a) None
b) A few in the T-zone (forehead and nose) only
c) Many
d) Tons!
e) Don't know (Note: please look again and only answer "e” if you cannot determine this.)

6. You would characterize your facial skin as:
a) Dry
b) Normal
c) Combination
d) Oily

7. When you use soap that suds, bubbles, and foams vigorously, your facial skin:
a) Feels dry, or cracks
b) Feels slightly dry but does not crack
c) Feels normal
d) Feels oily
e) I do not use soap or other foaming cleansers (If this is because they make your skin dry, pick "a.")

8. If not moisturized, your facial skin feels tight:
a) Always
b) Sometimes
c) Rarely
d) Never

9. You have clogged pores (black heads or white heads):
a) Never
b) Rarely
c) Sometimes
d) Always

10. Your face is oily in the "T-zone" (forehead and nose)
a) Never
b) Sometimes
c) Frequently
d) Always

11. Two to three hours after applying moisturizer your cheeks are:
a) Very rough, flaky or ashy
b) Smooth
c) Slightly shiny
d) Shiny and slick; or I do not use moisturizer 
Table 2. Questions with highest correlation with sebumeter measurement.

1. After washing your face, don't apply any moisturizer, sunscreen, toner, powder, or other products.

Two to three hours later, look in a mirror under bright lights. Your forehead and cheeks feel or appear:

a) Very rough, flaky, or ashy

b) Tight

c) Well hydrated with no reflection of light

d) Shiny with reflection of bright light

2. You would characterize your facial skin as:
a) Dry
b) Normal
c) Combination
d) Oily

3. Your face is oily in the T-zone (forehead and nose):
a) Never
b) Sometimes
c) Frequently
d) Always

4. You have clogged pores (blackheads or whiteheads):
a. Never
b. Rarely
c. Sometimes
d. Always

5. Your face is oily in the T-zone (forehead and nose):
a) Never
b) 5 or more hours after washing
c) 2 - 4 hours after washing
d) 1 hour after washing
e) All day

6. How many times a day do you wash your face?
a) I do not wash my face every day
b) 1 time
c) 2 times
d) 3 times
e) 4 or more

Table 3. Demographic characteristics of initial pilot test participants.

\begin{tabular}{cc}
\hline Demographic Characteristics & N (\%) \\
Age & \\
$\leq 45$ & $220(55.6)$ \\
$>46$ & $176(44.4)$ \\
Gender & \\
Male & $108(27.0)$ \\
Female & $292(73.0)$ \\
Race & \\
White Non-Hispanic & $174(43.6)$ \\
African American & $63(15.8)$ \\
Hispanic & $136(34.1)$ \\
Asian & $26(6.5)$ \\
\hline
\end{tabular}

gy Clinic at the Edelman Building of the University of Miami medical campus. Two trained interviewers were assigned to intercept all clinic patients as they entered the waiting room of the clinic. Participation in the survey was entirely voluntary. Informed consent was obtained before the survey was given to the respondents. Once 
enrolled, demographic information and a dermatological history were obtained by the two interviewers. Next, the study participants were given the survey and asked to complete the Baumann Skin Type Indicator (BSTI) questionnaire, version 1 [4]. The 11 items measuring skin oiliness were contained within the first section of the BSTI. Females were much more likely to complete the survey than males. The racial composition of the sample was predominantly White, non-Hispanic and Hispanic (Table 3). The surveys were collected, prior to the participants regularly scheduled dermatology consult performed by an expert dermatologist. The collected data were entered into an SPSS file. The present study was approved by the Institutional Review Board (IRB) and Ethics Committee of the University of Miami. This study was conducted according to all the Declaration of Helsinki Principles. Participants or their legal guardians gave their written informed consent.

The validation study conducted on the final six-item SOS involved 100 participants. The racial composition of the sample was predominantly White non-Hispanic and Hispanic females (Table 4). The surveys were collected prior to the participants regularly scheduled dermatology consult performed by an expert dermatologist. The collected data were entered into an SPSS file. Sebumeter measurements were taken from the chin in most cases, and in two bearded males from the forehead respectively at 45 minutes and 60 minutes.

\subsection{Statistical Analysis}

Descriptive statistical analyses of sample demographic information were conducted using SPSS, as was calculation of the corrected item-total correlation coefficient and Chronbach's alpha as an estimate of SOS score reliability. The partial credit model analyses used for the initial sample of 400 individuals responding to the initial bank of 11 items were conducted using Winsteps [10].

\section{Results}

A six-item SOS was developed (Table 5) and validated using standard measurement procedures, as outlined in Crocker and Algina, and Osterlind [5] [6]. A final validation and reliability study using a sample of 100 individuals was conducted to establish whether the SOS provides reliable and valid measures of skin oiliness. The obtained mean and standard deviation for each of the six items are also presented in Table 5.

Criterion-related evidence of validity for the SOS scores was obtained through consideration of the correlation between the SOS scores and the average of six sebumeter readings taken at 45 minutes and 60 minutes (three at each time point) post face-washing. The resulting correlation was 0.54 , which was significantly different from zero $(p<0.01)$. This result indicates a strong relationship between the SOS scores and the associated sebumeter measurements. A scatterplot showing the bivariate distribution of the SOS scores and the average sebumeter readings is shown in Figure 1.

Table 4. Demographic characteristics of validation study participants.

\begin{tabular}{cc}
\hline Demographic Characteristics & N (\%) \\
\hline$\leq 45$ & $29(29.0 \%)$ \\
$>46$ & $71(71.0 \%)$ \\
Gender & \\
Male & $4(4.0)$ \\
Female & $96(96.0)$ \\
Race & \\
White Non-Hispanic & $58(58.0)$ \\
African American & $3(3.0)$ \\
Hispanic & $37(37.0)$ \\
Asian & $2(2.0)$ \\
\hline
\end{tabular}




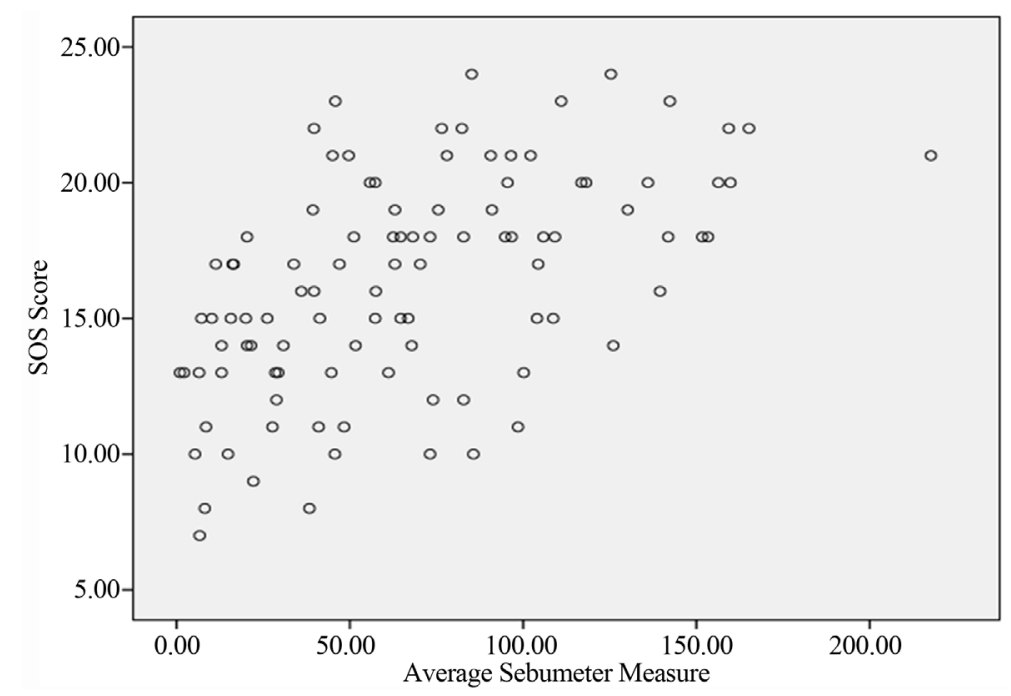

Figure 1. A scatterplot displaying the bivariate distribution of SOS scores and average sebumeter score.

Table 5. Mean and standard deviation of the responses to the SOS.

\begin{tabular}{lcc}
\hline \multicolumn{1}{c}{ Item } & Mean & SD \\
\hline 1. After washing your face... your forehead and cheeks feel or appear & 2.59 & 0.97 \\
2. You would characterize your facial skin as $\cdots$ & 2.59 & 0.94 \\
3. How frequently is your face oily in the T-zone? & 2.50 & 0.95 \\
4. How often do you have clogged pores? & 2.98 & 0.94 \\
5. How soon is your face oily in the T-zone after washing? & 2.70 & 1.33 \\
6. How many times a day do you wash your face? & 2.98 & 0.51 \\
\hline
\end{tabular}

a. Baumann skin type questionnaire (Part One: Oily vs. Dry).

Internal structure evidence of validity was obtained through consideration of the corrected item-total correlation coefficients for each item. The values of the corrected item-total correlation exceeded 0.45 for all items, with the exception of item 6 for which the value was 0.24 . The relatively low discrimination of item 6 was likely attributable to the small variability in the responses $(\mathrm{SD}=0.51)$ to the item (i.e. most responded as washing their face two times per day). Despite the seemingly low discrimination of item 6, its inclusion in the SOS was deemed to be important in order for the SOS to yield scores that would distinguish between individuals with moderately oily and very oily skin. Reliability of the obtained SOS scores was estimated using Cronbach's alpha, which yielded a value of 0.78 . This level of reliability is within acceptable levels for low-stakes scales of this nature, and ensures that the SOS provides consistent measures of skin oiliness.

\section{Discussion}

The results presented in Table 4 and Figure 1 show that the SOS scores are as valid as sebumeter scores to help determine skin oiliness. Patients with oily skin may have an increased incidence of acne, a decreased incidence of dry skin, and due to large amounts of vitamin E in sebum, may have reduced rates of skin cancer and photoaging. For these reasons, an easy to administer questionnaire to accurately determine skin oiliness can be useful in screening and recruiting patients for research trials, performing outcome research, and recommending skin care products and procedures. The SOS provides an inexpensive mechanism for measuring not just whether an individual's skin is oily, but also how oily the skin is. It can be clearly seen that SOS scores can provide an accurate qualitative measure of skin oiliness. 
Studies cited above have shown that asking patients if they have dry, oily or normal skin, does not correspond with sebumeter ratings. We have shown that a series of methodically designed questions does indeed correlate with sebumeter measurements. The questions must be used exactly as seen above in Table 1 in order to be valid, as other versions of the questions that have been studied over the last 5 years have been shown to have less validity. Similar studies that have developed oily skin questionnaires have included different questions from those of the SOS. For instance, the oily skin questionnaire developed by Segot-Chicq et al. [11] focuses on people's perception, behavioral and emotional circumstances about their skin oiliness. The SOS, in contrast, tries to avoid preconceptions about whether someone's skin is oily or dry and presents more in-depth questions that are specific to the degree of oiliness. It allows patients to select multiple choice answers that are appropriate for each scenario and provides detailed and accurate information about the most predominant skin oiliness factors.

\section{Conclusion}

In the past, questions evaluating a patient's subjective perception of skin oiliness were not found to correlate with sebumeter measurements. In many instances, it is important to determine a patient's level of sebum secretion, and a sebumeter is not always available. The findings of this study indicate that the six oily skin directed questions of the BSTI have the ability to classify individuals based on levels of sebum secretion. This can be used to recommend skin care products, to provide data in research trials and to divide patients into distinct groups based on sebum production in situations where a sebumeter is not accessible.

\section{Acknowledgements}

None.

\section{References}

[1] Kim, M.K., Choi, S.Y., Byun, H.J., Huh, C.H., Park, K.C., Patel, R.A., Shinn, A.H. and Youn, S.W. (2006) Comparison of Sebum Secretion, Skin Type, ph in Humans with and without Acne. Archives of Dermatologic Research, 298, 113-119. http://dx.doi.org/10.1007/s00403-006-0666-0

[2] Youn, S.W., Kim, S.J., Hwang, I.A. and Park, K.C. (2002) Evaluation of Facial Skin Type by Sebum Secretion: Discrepancies between Subjective Descriptions and Sebum Secretion. Skin Research and Technology, 8, 168-172. http://dx.doi.org/10.1034/j.1600-0846.2002.10320.x

[3] Youn, S.W., Na, J.I., Choi, S.Y., Huh, C.H. and Park, K.C. (2005) Regional and Seasonal Variations in Facial Sebum Secretions: A Proposal for the Definition of Combination Skin Type. Skin Research and Technology, 11, 189-195. http://dx.doi.org/10.1111/j.1600-0846.2005.00119.x

[4] Baumann, L. (2005) The Skin Type Solution. Bantam Dell, New York, 337.

[5] Crocker, L. and Algina, J. (1986) Introduction to Classical and Modern Test Theory. Harcourt, New York, 527.

[6] Osterlind, S.J. (2006) Modern Measurement: Theory, Principles, and Applications of Mental Appraisal. Pearson, Upper Saddle River, 492.

[7] Masters, G.N. (1982) A Rasch Model for Partial Credit Scoring. Psychometrika, 47, 149-174. http://dx.doi.org/10.1007/BF02296272

[8] Bond, T.G. and Fox, C.M. (2001) Applying the Rasch Model: Fundamental Measurement in the Human Sciences. Erlbaum, Mahwah, 255.

[9] Ebretson, S.E. and Reise, S.P. (2000) Item Response Theory for Psychologists. Erlbaum, Mahwah, 371.

[10] Linacre, J.M. (2005) A User’s Guide to WINSTEPS/MINISTEP Rasch-Model Computer Programs (Version 3.55). MESA Press, Chicago, 286.

[11] Segot-Chicq, E., Compan-Zaouati, D., Wolkenstein, P., Consoli, S., Rodary, C., Delvigne, V., Guillou, V. and Poli, F. (2007) Development and Validation of a Questionnaire to Evaluate How a Cosmetic Product for Oily Skin Is Able to Improve Well-Being in Women. Journal of the European Academy of Dermatology and Venereology, 9, 1181-1186. http://www.ncbi.nlm.nih.gob/pubmed/17894702 